\title{
Buchbesprechungen - Book Reviews - Livres Nouveaux
}

M. Plotz: Coronary Heart Disease. Angina Pectoris, Myocardial Infarction. Preface by William Bock. Paul B. Hoeber, New York 1957. XI + 353 p., num. fig. \$12.00.

Der Autor hat in seinem Buch die heutige Auffassung über die Angina pectoris in sehr klarer, erschöpfender und anregender Art vorgelegt. Es sind alle Gesichtspunkte, die Arzte und Laien bei dieser menschlichsten Kranklieit (wie W. Dock sagt: da sie niemals bei Tieren spontan beobachtet wurde) be-

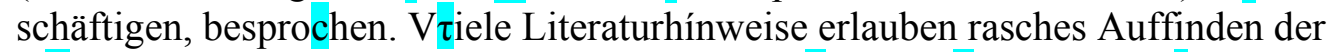
Originalarbeiten. Das Buch ist daher besonders für die Bedürfnisse der Internisten und Kardiologen vom praklischen Standpunkt aus sehr geeignet und kann sehr empfohlen werden. R. H.

W. Teschendorf: Lehrbuch der rön $1 / 8$ enologischen Differentialdia $7 / 8$ nostik. Band I. Erkrankungen der Brustorgane. 4. verbesserte und erweiterte Aufl. G. Thieme, Stuttgart 1958. XII + 1183 S., 1138 Abb. DM 210.-.

Die 4. Auflage des bekannten und bewährten Buches von Teschendorf hat eine wesentliche Umgestaltung erfahren. Das große Kapitel der Herzkrankheiten hat Thurn geschrieben und in jeder Beziehung auf den neuesten Stand gebracht, Bei der durch die Einführung neuer Untersuchungsmethoden (Angiokardiographie und Herzkatheterismus) erreichten, großen Erweiterung der Kenntnisse auf diesem Gebiet erwies sich eine Umarbeilung des ganzen Kapitels als notwendig. Die vorliegende Arbeit gibt einen sehr vollständigen, aber auch kritischen Überblick. Das Buch eignet sich auch vorzüglich als Nachschlagewerk, da viele und gut ausgewählte Literaturhinweise angeführt sind. Die röntgenologischen Bilder werden stets mit pathologisch-anatomischen und pathophysiologischen Befunden zu erklären versucht. Das Werk kann jedem Internisten und Kardiologen bestens empfohlen werden. R. H.

Pr. F. Jourdan: Travaux de $\Gamma$ Institut de Recherches cardiologiques de Koyat. Vol. 111 (1952-1954). 278 p., 72 fig.

Ce recueil broché renferme 24 travaux scientifiques faits à Boyat, sous la direction du Pr. F. Jourdan, déjà publiés dans diverses revues de 1952 à 1956.

Le plus important d'entre eux est la these de $\mathrm{M}^{\mathrm{TM}} \mathrm{c}$ Jeanine Duchêne-Marullaz intitule : La médullo-surrénale au repos et en actwiité. (Test un gros travail experimental sur le Lapin, où $\Gamma$ on a étudié la teneur en principe vaso-constrícteur et $\Gamma$ intensité de la reaction chromaffine de la glande médullo-surrénale à $\Gamma$ état de repos et quand son activité sécrétoire est déclenchée par Гexcitation des nerfs splanchniques. Cette dernière réduit la teneur en principe vaso-constricteur de la glande sans modifier sa reaction chromaffine. II n'y a pas de relation entre Tintensité de cette reaction et la teneur en principe vaso-constricteur. Les deux glandes d'un même animal ont des reactions qualitatives et quanlitatives similaires.

Huit de ces travaux sont consacrés à $\Gamma$ étude expérimentale du tonus cardio-accélérateur et des reflexes baro-sensibles sous diverses influences. 
Buchbesprechungen - Book Reviews - Livres Nouveaux 331

Six autres étudient le rythme nodal experimental chez Tanimal et les rythmes nodaux spontanés chez l'homme.

Trois analysent Faction du gaz thermal de Royat sur la circulation céré-brale, sur les nerfs vasomoteurs et dans Tartérite oblilérante des membres.

Trois sont consacrés aux effets du Largactil sur le système nerveux végéta-tif, les centres cardioaccélérateurs et sur la glande médullo-surrénale.

Deux travaux de P. Bailliart étudient la vaso-motricité et la regulation de la circulation

rétínienne.

L'un, publié avec R. Froment, est une recherche expérimentale sur les correlations physio-

pathologiques neuro- et viscérocoronariennes.

Cette liste porte à 61 le total des travaux de $\Gamma$ lnstitut de recherches cardio-

logiques, ce qui est tout à Thonneur de Гimportante activité scientifique expéri

mentale et clinique qui se développe à la station balnéaire de Royat, sous la

direction du Pr. F. Jourdan. Ch. Mahaim, Lausanne.

M. Faucon: L'inhalation d'oxygène chez les cmphysémateux au stade du cæur pulmonaire

chronique. Son effet sur la ventilation et la circulation pulmonaires. These (Hôpital Saint-Joseph, Lyon). $99 \mathrm{p}$.

Der Autor berichtet sehr ausführlich über den Einfluß der 02-Atmung bei 27

Emphysempatienten, von denen 13 an einem chronischem Cor pulmonale litten. Untersucht

wurden die arteriellen Blutgase (02-Sttg., C02-Gehalt und -spannung, $\mathrm{pH}$ ), das

Herzminutenvolumen und die Ventilation. Er bestätigt einmal mehr die Reduktion der

Ventilation in Fallen mit vorbestehender alveolärer Hypoventilation und damit die Verstärkung der respiratorischen Acidose durch 02-Atmung in diesen Fallen. Bei Almung von 4õ\% 02 kommt es zu einem leichten Druckabfall in der Art. pulmonalis, die Änderungen des

Herzminutenvolumens sind unterschiedlich. Bei $10 C \% 02$ kam es in Einzel-fällen mit einer deutlichen zusätzlichen C02-Retention zu einem leichten Druckanstieg. Während der Druck im Körperkreislauf bei 02-Atmung nicht signifikant ändert, ist bei Rückatmungsversuchen mit einer massiven C02-Retention ein Druckanstieg im Lungen- und Körperkreislauf zu beobachten. Mit künstlicher Steigerung der Ventilation mittels eines Tankrespirators kommt es mit der Besserung der Blutgase, insbesondere der C02-Spannung zu einem leichten Druckabfall im

Lungenkreislauf. Auf Grund dieser Befunde halt der Autor die 02-Therapie bei

Emphysempatienten nur in Kombination mit ventilationssteigernden Maßnahmen, z. B. auch Atemgymnastik, für sinnvoll.

A. Bühlmann, Zurich.

Kaufmann-Staemmler: Lehrbuch d. spez. patholog. Anatomic. Bd. 111, Lfg. 3. 11. u. 12. Aufl.

272-426 Seiten. Walter de Gruyter, Berlin 1958. Subskr.Pr. DM 28.-.

Das vorliegende neueste wohlgelungene Heft befaßt sich mit den sehr wichtigen

Kreislaufstörungen und Gefäí.îerkrankungen des Zentralnerven-systems (Staemmler), wobei

auch das Problem des Hirnödems und der Hirn-schwellung zur Sprache kommen. Ein zweites

Kapitel ist den Fehlbildungen des Zentralnervensystems gewidmet (G. Peter und O. Lund).

Beide Abschnitte fügen sich sehr gut in den allgemeinen Rahmen dieses wertvollen Lehrbuches

für den Spezialisten und Praktiker ein. Der Text ist sehr kurz gefaßt mit guten

332 Buchbespreehungen - Book Reviews - Livres Nouveaux

Schrífttumhinweisen, die Abbildungen sind praktisch sämtliche neu, und beim

Durchblättern der dem Ref. besonders heimischen Kapitel stellt er mit Freude 
fest, daß auch die neuesten Auffasungen und Erkenntnisse berücksichtigt wurden. H. U. Zollingcr, St. Gallen.

Rossier, P. H., A. Bühlmann und K. Wiesinger: Physiologic und Pathophysio-logic der Atmung. 2., verbesse te und erweiterte Auflage. Springer-Verlag, Berlin 1958. VIII +395 Seiten, $9 \delta$ Abbildungen. DM 58.60.

Das bekannte Buch von Rossier und Bühlmann, welches die Ergebnisse einer jahrzehntelangen systematisehen Arbeit über die Probleme der Lungenfunktion der Zürcher Schule zusammenfal.it, ist in zweiter Auflage mil mannigfachen Ergänzungen und Erweiterungen erschienen. Für die Kardiologen von Bedeutung ist die erschöpfende und kritische Darstellung der engen Beziehungen zum Kreislauf. Das Buch bildet für alle, die gieh mit diesen Pioblemen beschäftigen, eine Fülle von Anregungen und ist auch als Nachschlagewerk unentbehrlich. R. H. J. Jacobi und M. Loeweneck: Operable Herzleiden. Einführung in Klinik, Dia-gnostik und Operationsmöglichkeiten. Enter Mitarbeit von K. Metier und H. Samlert. G. Thieme, Stuttgart 1958. VIII + 175 Seiten, 155 Abbildungen in 268 Einzeldarstellungen. DM 49.50. Die Autoren haben es in ihrem Buch unternommen, das Gebiet der ope rablen Herzleiden, welches in den letzten Jahren bekanntlich durch den Herz-katheterismus und die Operationsmöglichkeiten eine enorme Erweiterung er-fahren hat, für die Bedürfnisse der vorwiegend praktisch tätigen Kardiologen und Arzte darzustellen. Man darf sagen, daß ihnen dieser Versuch vorzüglich gelungen ist. Alle, welche nicht über eine eigene größere Erfahrung verfügen, werden aus der Darstellung der beiden Hamburger großen Nutzen ziehen.

R.H.

Varia.

Le bureau de la Société Française de Cardiologie a Thonneur d'annoncer la creation de la Société Française pour $\Gamma$ Etude de $\Gamma$ Athérosclérose. Celle-ci sera une section de la Société Française de Cardiologie.

The Second Asian/Pacific Congress of Cardiology will be held in Melbourne, Australia, during the last week in May, 1960. Further deta:ls may be obtained from Dr. A. E. Doyle, Honorary Secretary, Alfred Hospital, Melbourne, S. 1, Victoria, Australia.

Erratum.

Das Klischee zur Abbildung 1/2 der Arbeit Pauli, Heft 1, Seite 64, dieses Bandes der CARDIOLOGIA steht auf dem Kopf. Es ist also um $180^{\circ} \mathrm{zu}$ drehen, die bisherige Abbildung 1 ist in Wirklichkeit Abbildung 2, und die bisherige Abbildung 2 ist tatsächlich Abbildung 1. 